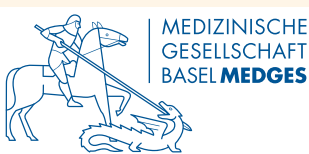

Gemäss den medizinisch-ethischen Richtlinien der Schweizerischen Akademie für medizinische Wissenschaften SAMW aus dem Jahr 2004 ist «die Beihilfe zum Suizid nicht Teil der ärztlichen Tätigkeit, weil sie den Zielen der Medizin widerspricht». In Ärztekreisen mehren sich die Stimmen, die diese Haltung angesichts des intensiven gesellschaftlichen Diskurses, der zu diesem Thema in den letzten Jahren geführt wurde, für nicht mehr zeitgemäss halten. Allerdings stösst die explizite Auffassung, Suizidhilfe sei als Teil der ärztlichen Aufgaben zu sehen, innerhalb der Ärzteschaft auch auf dezidierten Widerspruch.

\section{Diskutieren Sie mit}

Mit dem Basler Podium möchte die Schweizerische Ärztezeitung zu einer sachlichen und differenzierten Auseinandersetzung mit den Fragen beitragen, die die Ärzteschaft im Zusammenhang mit ärztlicher Suizidhilfe beschäftigen. Der Einbezug des Publikums in die Diskussion ist zentraler Bestandteil des Konzepts der SÄZ-Podiumsveranstaltungen.

\section{Datum / Zeit / Ort}

Die Podiumsveranstaltung mit anschliessendem Apéro findet statt am Dienstag, 27. November, 18.3020.30 Uhr, im BaZ City Forum am Aeschenplatz, Dufourstrasse 49, Basel.

\section{Anmeldung}

Der Eintritt zur Veranstaltung ist frei, eine Anmeldung ist aber erforderlich. Anmeldungen können bis Freitag, 23. November via E-Mail an redaktion. saez[at]emh.ch oder via Fax an 0614678556 erfolgen. Bitte Ihren Namen und die Namen allfälliger Begleitpersonen sowie das Stichwort «Anmeldung zum SÄZ-Podium vom 27. November» angeben. Auch telefonische Anmeldungen sind vormittags unter 0614678572 möglich.

\title{
Die Podiumsgäste
}

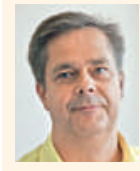

Eberhard Wolff

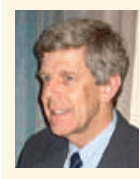

Klaus

Bally

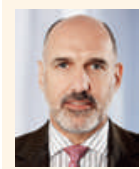

Jacques de Haller
Unter der Leitung von PD Dr. rer. soc. Eberhard Wolff, Redaktor Medizingeschichte der Schweizerischen Ärztezeitung und Lehrbeauftragter an den Universitäten Zürich und Basel, diskutieren:

Dr. med. Klaus Bally, Hausarzt und Lehrbeauftragter am Institut für Hausarztmedizin der Universität Basel

Dr. med. Jacques de Haller, Präsident der FMH

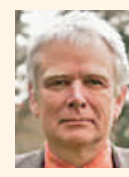

Johannes Fischer

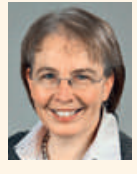

Marion

Schafroth

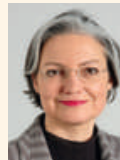

Gabriela Stoppe
Prof. Dr. theol. Johannes Fischer, ehem. Mitglied der Zentralen Ethikkommission und beigezogener Experte bei der Ausarbeitung der SAMWRichtlinien zur Betreuung von Patienten/-innen am Lebensende

Dr. med. Marion Schafroth, Fachärztin für Anästhesiologie FMH und Vorstandsmitglied EXIT, Ressort Freitodbegleitung

Prof. Dr. med. Gabriela Stoppe, Fachärztin für Psychiatrie und Psychotherapie FMH, Leiterin der AG Mental Health und alternde Bevölkerung, Swiss Public Health 